OPEN ACCESS

Edited by:

Awdhesh Kalia,

University of Texas MD Anderson Cancer Center, United States

Reviewed by:

Víctor Antonio García-Angulo, Universidad de Chile, Chile Anthony Flores,

McGovern Medical School, United States

${ }^{*}$ Correspondence: Nadja Patenge nadja.patenge@med.uni-rostock.de

${ }^{\dagger}$ Present address: Nicole Popp,

BD Life Sciences, Heidelberg,

Germany

Specialty section:

This article was submitted to Infectious Diseases,

a section of the journal

Frontiers in Microbiology

Received: 14 November 2017

Accepted: 29 January 2018

Published: 20 February 2018

Citation:

Khani A, Popp N, Kreikemeyer B and Patenge N (2018) A Glycine Riboswitch in Streptococcus pyogenes Controls Expression of a Sodium:Alanine Symporter Family Protein Gene. Front. Microbiol. 9:200. doi: 10.3389/fmicb.2018.00200

\section{A Glycine Riboswitch in Streptococcus pyogenes Controls Expression of a Sodium:Alanine Symporter Family Protein Gene}

\author{
Afsaneh Khani, Nicole Popp', Bernd Kreikemeyer and Nadja Patenge* \\ Institute of Medical Microbiology, Virology and Hygiene, University Medicine Rostock, Rostock, Germany
}

Regulatory RNAs play important roles in the control of bacterial gene expression. In this study, we investigated gene expression regulation by a putative glycine riboswitch located in the $5^{\prime}$-untranslated region of a sodium:alanine symporter family (SAF) protein gene in the group A Streptococcus pyogenes serotype M49 strain 591. Glycinedependent gene expression mediated by riboswitch activity was studied using a luciferase reporter gene system. Maximal reporter gene expression was observed in the absence of glycine and in the presence of low glycine concentrations. Differences in glycine-dependent gene expression were not based on differential promoter activity. Expression of the SAF protein gene and the downstream putative cation efflux protein gene was investigated in wild-type bacteria by RT-qPCR transcript analyses. During growth in the presence of glycine $(\geq 1 \mathrm{mM})$, expression of the genes were downregulated. Northern blot analyses revealed premature transcription termination in the presence of high glycine concentrations. Growth in the presence of $0.1 \mathrm{mM}$ glycine led to the production of a full-length transcript. Furthermore, stability of the SAF protein gene transcript was drastically reduced in the presence of glycine. We conclude that the putative glycine riboswitch in S. pyogenes serotype M49 strain 591 represses expression of the SAF protein gene and the downstream putative cation efflux protein gene in the presence of high glycine concentrations. Sequence and secondary structure comparisons indicated that the streptococcal riboswitch belongs to the class of tandem aptamer glycine riboswitches.

Keywords: glycine, riboswitch, Streptococcus pyogenes, cis-regulatory element, amino acid riboswitch, regulatory RNAs, transcription genetic

\section{INTRODUCTION}

Bacterial riboswitches are cis-regulatory elements found in the $5^{\prime}$-untranslated regions (UTRs) of mRNAs. Regulation of gene expression by riboswitches is widespread in bacteria. In general, aptamer structures within the riboswitch recognize small molecule ligands and interact with an expression platform. Ligand binding promotes formation of an exclusive conformation and leads to the regulation of downstream genes. Repression of gene expression is achieved by transcription termination, inhibition of translation via sequestration of the ribosome binding site, or mRNA processing. Binding of ligands which induce gene expression, allows transcription elongation 
or translation initiation. Riboswitches from different classes recognize a large range of cellular compounds, including coenzymes (Winkler W. et al., 2002; Winkler W.C. et al., 2002; Winkler et al., 2003), magnesium cations (Dann et al., 2007), purines and their derivatives (Batey, 2012), second-messenger molecules (Sudarsan et al., 2008), and amino acids (Serganov and Patel, 2009). In many cases, riboswitches control the expression of genes that are involved in the biosynthesis, degradation, or transport of the respective metabolite.

Bacteria depend on the availability of amino acids for continuous protein biosynthesis. Therefore, expression of genes required for amino acid transport, synthesis, and degradation is tightly regulated. Typical sensors for amino acid levels are T-box elements and attenuators located in the mRNA. T-box RNA binds tRNAs and modulates transcription or translation of the gene under control (Grundy and Henkin, 1993). Attenuators contain codons for an effector amino acid. At a low concentration of the effector, translation stalls and formation of an anti-terminator structure is favored (Yanofsky, 1981). In addition, two riboswitches are able to bind amino acids directly. Lysine riboswitches control genes involved in lysine biosynthesis and lysine transport (Grundy et al., 2003; Rodionov et al., 2003; Sudarsan et al., 2003). Glycine riboswitches most often regulate the expression of glycine cleavage system genes, but were also found upstream of various other genes involved in the synthesis, conversion, or transport of glycine (Barrick et al., 2004). Glycine riboswitches are widely distributed and have been identified in Gram-positive and Gram-negative bacteria (Barrick and Breaker, 2007).

Streptococcus pyogenes [Group A streptococcus (GAS)] is a Gram-positive human pathogen responsible for a variety of diseases ranging from mild self-limiting superficial infections of the throat or skin to life-threatening invasive diseases including bacteremia and necrotizing fasciitis. Common post-streptococcal autoimmune complications are acute rheumatic fever and acute post-streptococcal glomerulonephritis. The impact of GAS diseases is especially high in resource-limited settings and in a recent publication a rise of global invasive disease burden caused by GAS was reported (Sims et al., 2016).

To limit the application and distribution of antibiotics and to identify GAS specific therapeutic targets, investigation of metabolic regulation processes can be very fruitful. Riboswitches have been already studied as promising antibacterial targets in a variety of bacteria. Modification of riboswitch function is achieved by the administration of ligand analogs, usually small compounds which are easy to manufacture and to deliver (Machtel et al., 2016). Glycine supplementation is required for optimal growth of GAS (Levering et al., 2016). The presence of a putative glycine riboswitch in GAS opens the possibility to specifically inhibit glycine metabolism or transport. Therefore, we investigated in this study gene expression regulation by a cisregulatory element with sequence similarities to known glycine riboswitches. One glycine binding aptamer was predicted in the $5^{\prime}$-UTR of a sodium:alanine symporter family protein (SAF) gene in GAS M49 strain 591. We found that the genetic element indeed regulates glycine-dependent gene expression of the SAF gene by transcription termination/anti-termination. On a second level of regulation, SAF gene transcript stability is reduced in the presence of glycine.

\section{MATERIALS AND METHODS}

\section{Bacterial Strains and Culture Conditions}

Group A streptococcus serotype M49 strain 591 was kindly provided by R. Lütticken (Aachen, Germany). All GAS strains were cultured in chemically defined medium (CDM) (van de Rijn and Kessler, 1980) or Todd-Hewitt broth (Thermo Fisher Scientific, Darmstadt, Germany) supplemented with $0.5 \%$ yeast extract (Thermo Fisher Scientific, Darmstadt, Germany) (THY), as indicated, at $37^{\circ} \mathrm{C}$ with a $5 \% \quad \mathrm{CO}_{2} / 20 \% \quad \mathrm{O}_{2}$ atmosphere. Escherichia coli strain DH5 $\alpha$ (Gibco BRL, Eggenstein, Germany) was used as host for the construction, proliferation, and storage of recombinant plasmids. All E. coli strains were cultured in Lennox L Broth Base (Thermo Fisher Scientific, Darmstadt, Germany). For selection, antibiotics were added at the appropriate concentrations.

\section{Construction of Recombinant GAS Strains}

Riboswitch controlled LUC reporter gene constructs were generated by cloning an 875 bp genomic fragment into the MCS of pFW11-luc2 (Podbielski et al., 1999). Promoter controlled LUC reporter gene constructs were generated by fusing a 588 bp fragment carrying the promoter region $5^{\prime}$ of ribogly to luc2. Inserts were amplified by PCR using chromosomal DNA from GAS M49 strain 591 as template. All primers used for the generation of the respective fragments are listed in Supplementary Table 1 . The resulting plasmids were verified by classical Sanger sequencing (GATC Biotech AG, Konstanz, Germany). GAS M49 strain 591 was transformed with the respective plasmids. Insertion of the recombinant fusion genes into the genome was verified by PCR analyses and classical Sanger sequencing (GATC Biotech AG, Konstanz, Germany) of a genomic PCR fragment.

\section{Quantitative Assay for Luciferase Activity}

For assessment of luciferase activity from riboswitch-luc fusions, GAS luc reporter strains were grown in CDM, supplemented with $0-10 \mathrm{mM}$ of the amino acids glycine, alanine, or serine as indicated. For measurement of luminescence, $1 \mathrm{ml}$ aliquots of the cell suspensions were withdrawn at hourly intervals, $\mathrm{OD}_{600}$ was measured, and samples were processed as described by Podbielski et al. (1999). Luminescence was measured for $15 \mathrm{~s}$ in a Luminometer Lumimat LB 9501 (Berthold Technologies $\mathrm{GmbH}$, Bad Wildbad, Germany). RLU values at each time point were calculated by subtracting luminescence at time 0 from luminescence at time $\mathrm{x}$. RLU/OD 600 were calculated to normalize for growth differences.

\section{Extraction of Total RNA}

Group A streptococcus strains were grown for $3 \mathrm{~h}$ in CDM complemented with glycine as indicated. Bacteria were pelleted 
immediately, quickly frozen in liquid nitrogen, and stored at $-80^{\circ} \mathrm{C}$ until use. Bacterial cells were disrupted in a homogenizer (Peqlab Biotechnologie GmbH, Erlangen, Germany). Total RNA from GAS strains was extracted according to the protocol supplied with the Direct-zol ${ }^{\mathrm{TM}} \mathrm{RNA}$ MiniPrep Kit (Zymo Research, Irvine). After extraction, RNA was treated with acid phenol:chloroform:isoamyl alcohol (125:24:1), pH 4.5 (Thermo Fisher Scientific, Darmstadt, Germany), and TURBO ${ }^{\mathrm{TM}}$ DNAse (Thermo Fisher Scientific, Darmstadt, Germany) according to the manufacturer's instructions. RNA was stored at $-80^{\circ} \mathrm{C}$ until further use.

\section{Reverse Transcription Followed by Quantitative PCR (RT-qPCR)}

Following DNAse treatment, cDNA synthesis was performed using the Superscript first-strand synthesis system for RT-PCR (Invitrogen, Thermo Fisher Scientific, Darmstadt, Germany). Quantitative PCR amplification was conducted with SYBR green (Thermo Fisher Scientific, Darmstadt, Germany) using the ViiA ${ }^{\mathrm{TM}} 7$ Real-Time PCR System (Applied Biosystems, Darmstadt, Germany). The 5S rRNA gene served as internal control. Relative expression was calculated employing the $2^{-\Delta \Delta}$ ct method (Schmittgen and Livak, 2008). All primers used for RT-qPCR are listed in Supplementary Table 1.

\section{Northern Blot Analyses}

RNA samples $(6 \mu \mathrm{g})$ were loaded onto a denaturing $1.5 \%$ formaldehyde agarose gel and separated by electrophoresis. Size standards (Millennium ${ }^{\mathrm{TM}}$ RNA Marker; Thermo Fisher Scientific, Darmstadt, Germany) were loaded on the same gel. RNA was blotted onto neutral nylon membranes (Roti ${ }^{\circledR}$-Nylon 0.2, Carl Roth GmbH \& co KG, Karlsruhe, Germany) and UV cross-linked (UV150 mJoule $/ \mathrm{cm}^{2}$ ). Templates for the probes were generated by PCR with the primers listed in Supplementary Table 1. The $5^{\prime}$ end of the reverse primers contained the $\mathrm{T} 7$ promoter sequence (CTTAATACGACTCACTATAGGG) for in vitro transcription using the MEGAscript ${ }^{\circledR}$ T7 Kit (Thermo Fisher Scientific, Darmstadt, Germany). Probes were labeled with biotin using the Pierce TM RNA 3' End Biotinylation Kit (Thermo Fisher Scientific, Darmstadt, Germany). Biotin labeled RNA probes were purified using the RNA Clean \& Concentrator ${ }^{\mathrm{TM}}-5$ kit (Zymo Research, Freiburg, Germany). Membranes were hybridized overnight with RNA probes complementary to the respective transcripts as indicated. IRDye 800CW Streptavidin (LI-COR Biotechnology $\mathrm{GmbH}$, Bad Homburg, Germany) was used for detection, and the blot was scanned on an Odyssey ${ }^{\circledR}$ Imager CLX 1283 (LI-COR Biotechnology GmbH, Bad Homburg, Germany).

\section{Transcript Stability Determination}

Group A streptococcus strains were grown for $3 \mathrm{~h}$ in CDM complemented with glycine as indicated. RNA synthesis was inhibited by addition of rifampicin $(1 \mathrm{mg} / \mathrm{ml})$ to the cultures. Following the addition of rifampicin, $5 \mathrm{ml}$ samples were recovered after $0,1,2,5$, and $10 \mathrm{~min}$. Samples were added to two volumes of RNAprotect Cell Reagent (Qiagen $\mathrm{GmbH}$, Hilden, Germany), incubated at room temperature for $5 \mathrm{~min}$, pelleted by centrifugation and quick frozen in liquid nitrogen. RNA was isolated from the samples as described above and transcript abundance was determined by RT-qPCR. The half-life of the transcripts was calculated by non-linear regression analyses (Least squares fit, GraphPad Prism).

\section{Statistical Analyses}

All experiments were performed at least three times or as indicated by the sample size (n). Statistical significance was determined using the tests indicated in the respective figure legends.

\section{RESULTS}

\section{The Putative Glycine Riboswitch Mediates Glycine-Dependent Expression of a Luciferase Reporter Gene}

In a previous study, we detected expression of 18 putative riboswitches using intergenic DNA tiling arrays (Patenge et al., 2012). One riboswitch candidate was identified downstream of pcrA (Spy49_1007c) and was designated sRNASpy491007c. It belongs to the Rfam family RF00504 of glycine riboswitches (Nawrocki et al., 2015) and is located in the 5-prime region of the sodium:alanine symporter family protein (SAF) gene (Reizer et al., 1994) (Figure 1). A luciferase (LUC) reporter gene system was employed to determine a potential response to glycine. An 889 bp genomic fragment containing the predicted glycine riboswitch sequence and, to allow for homologous recombination, its $5^{\prime}$ flanking genomic region was amplified by PCR and fused to the luc reporter gene carried by pFW11_luc2 (Podbielski et al., 1999) (Figure 1B). GAS M49 strain 591/pFW11_glyluc2 was grown in CDM containing varying concentrations of glycine. Glycine is required for optimal growth of GAS (Levering et al., 2016). Consistently, growth was poor in medium without glycine and in medium containing a low glycine concentration ( $0.01 \mathrm{mM}$ glycine) compared to growth in medium containing 0.1 to $10 \mathrm{mM}$ glycine (Figure 2A). LUC activity was measured over time and normalized to cell density to compensate for differences in growth (Figure 2B). Maximal reporter gene expression was observed following $3 \mathrm{~h}$ of growth in the absence of glycine and in the presence of low glycine concentrations (0.01-0.1 mM). In the presence of $10 \mathrm{mM}$ glycine, reporter gene expression was always repressed. During growth in $1 \mathrm{mM}$ glycine, moderate LUC activity could be detected in the exponential growth phase. Since the gene downstream of the putative glycine riboswitch encodes a putative sodium:alanine symporter and since alanine and serine are structurally similar to glycine, we tested the effect of these two amino acids on reporter gene expression. Growth of GAS M49 strain 591/pFW11_glyluc2 in the absence of serine and in CDM containing $0.01 \mathrm{mM}$ serine was slow compared to growth in the presence of $0.1-10 \mathrm{mM}$ serine (Figure 2C). GAS M49 strain 591/pFW11_glyluc2 grew similar in CDM with and without alanine (Figure 2E). Neither 


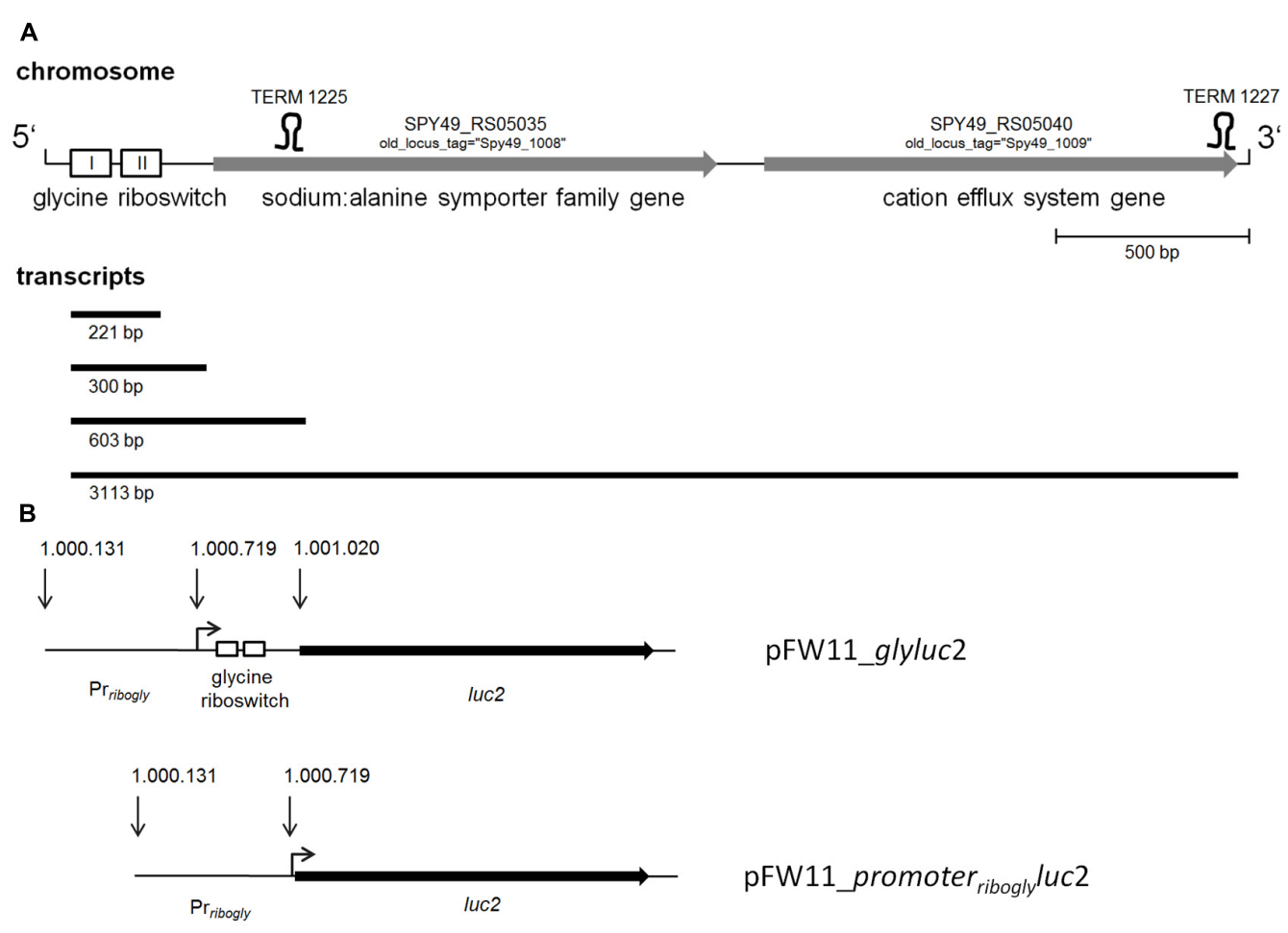

FIGURE 1 | (A) Schematic representation of the glycine riboswitch locus. Glycine riboswitch aptamers 1 and 2 are depicted as boxes I and II; genes are depicted as gray arrows, pointing into the direction of transcription; terminators 1225 and 1227 as predicted by TransTermHP (Kingsford et al., 2007) are drawn as stem-loop structures, and transcripts detected by Northern blotting (Figure 4) are depicted as black lines. (B) Schematic representation of the riboswitch-/uc2 fusion constructs: pFW11_glyluc2 consists of an 889 bp genomic fragment containing the predicted glycine riboswitch sequence and its $5^{\prime}$ flanking genomic region including the promoter region fused to luc2. pFW11_promoter ribogly luc2 consists of an 588 bp fragment including the promoter region $5^{\prime}$ of ribogly directly fused to luc2.

the presence of serine nor alanine did influence reporter gene expression (Figures 2D,F).

\section{The Putative Glycine Riboswitch Controls Expression the SAF Gene and a Putative Cation Efflux System Gene}

To address the question, whether the putative glycine riboswitch controls expression of its downstream genes, RT-qPCR experiments were conducted. WT bacteria were grown in CDM containing $0.1,2.6$, and $10 \mathrm{mM}$ glycine. Total RNA was isolated, reverse transcribed, and amplified using primers specific for the putative glycine riboswitch (ribogly), the SAF gene $\left(\mathrm{Na}^{+} / \mathrm{Ala}\right.$ symp), and the putative cation efflux system gene (cation efflux), respectively (Figure 3A). Transcript abundance at $0.1 \mathrm{mM}$ glycine served as calibrator for all three reactions. Abundance of the riboswitch RNA was unaltered at all glycine concentrations tested. In contrast, the SAF and the cation efflux system gene mRNA were dramatically decreased at 2.6 and $10 \mathrm{mM}$ glycine in comparison to $0.1 \mathrm{mM}$ glycine.

\section{Glycine-Dependent Gene Control Is Not Based on Changes of Promoter Activity}

To investigate the regulatory influence of the promoter region, a 588 bp fragment carrying the promoter region $5^{\prime}$ of ribogly was fused to luc (Figure 1B). GAS M49 strain 591 was transformed

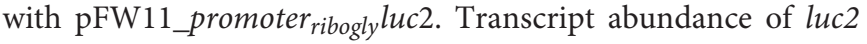
was determined by RT-qPCR following growth in the presence of $0.1,2.6$, and $10 \mathrm{mM}$ glycine. Transcript level at $0.1 \mathrm{mM}$ glycine served as calibrator. Expression of $l u c 2$ was not influenced by the glycine concentration in the medium (Figure 3B).

\section{Transcript Sizes Are Dependent on the Glycine Concentration in the Medium}

Expression of both, the SAF gene and the cation efflux system gene, was repressed in the presence of high glycine concentrations (Figure 3). Primers to amplify gene junctions were designed and used in reverse-transcriptase experiments followed by PCR. The detection of a PCR product suggested co-transcription of the two genes. To verify this result, Northern blot analyses were performed. WT bacteria were grown in CDM containing 0.1, 2.6, or $10 \mathrm{mM}$ glycine, respectively. Total RNA was isolated from the cultures and separated by agarose gel electrophoresis. Blots were sequentially hybridized with RNA probes specific for ribogly and the SAF gene transcript $\left(\mathrm{Na}^{+} /\right.$Ala symp). In the presence of high glycine concentrations $(2.6$ and $10 \mathrm{mM})$, the ribogly probe detected a highly abundant $200 \mathrm{bp}$ band, which might represent a transcription termination or a RNA cleavage product (Figure 4). No SAF gene transcript could be detected (Figure 4). Consistent 

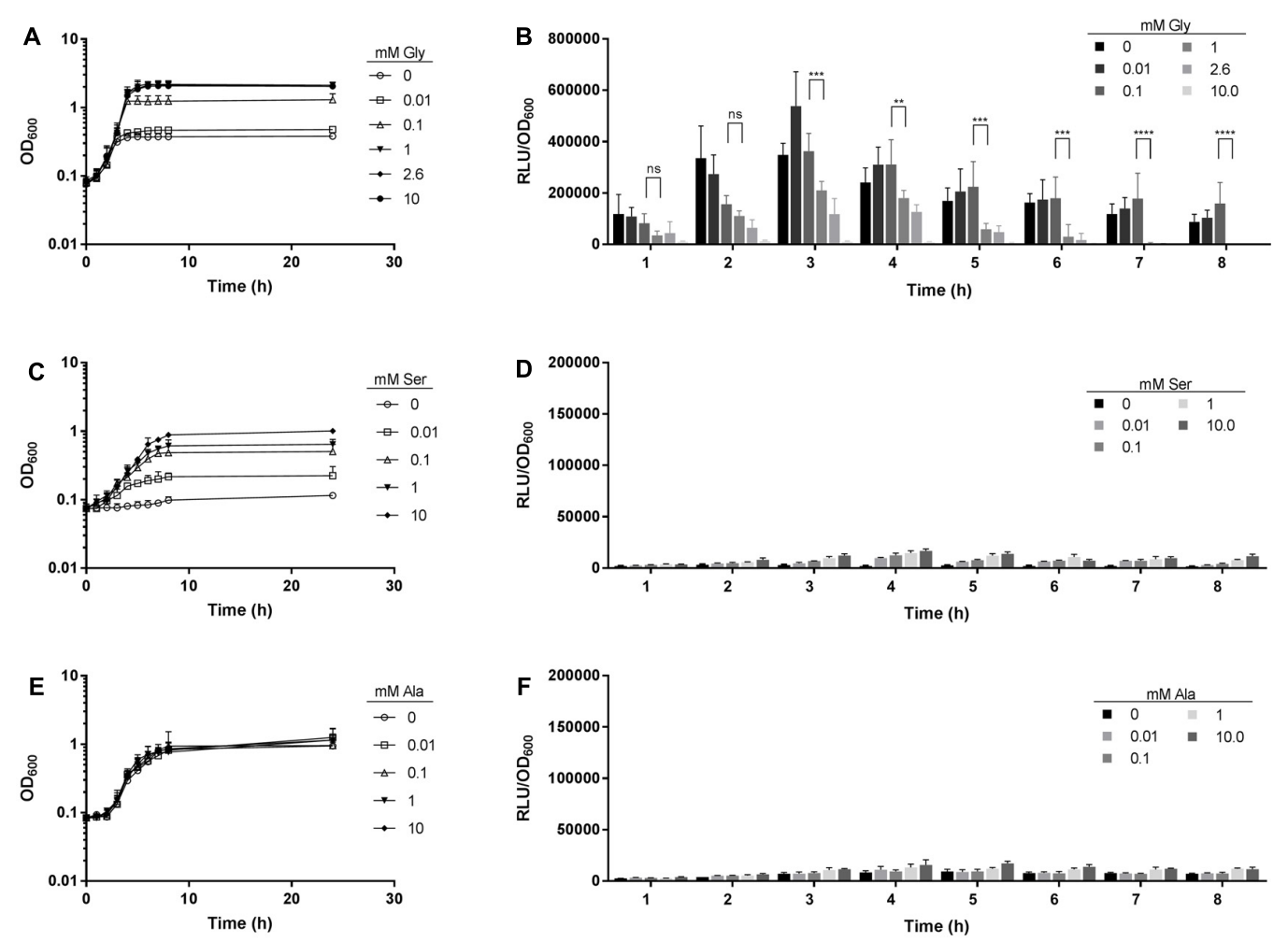

FIGURE 2 | LUC reporter gene expression is repressed at high glycine concentrations. (A) Growth of GAS M49 strain 591/pFW11_glyluc2 in CDM without glycine or supplemented with different concentrations (0.01-10 mM) of glycine, $n=5$. (B) Luciferase activity over time in GAS M49 strain 591/pFW11_glyluc2 during growth without glycine or in the presence of different concentrations of glycine, as indicated, $n=5$. Statistical significance was determined between 0.1 and $1 \mathrm{mM}$ glycine samples using the two-way ANOVA test (multiple comparisons). Differences between samples were expressed as "ns": not significant, $P \geq 0.05 ;{ }^{*} P<0.05$; ${ }^{* *} P<0.01 ;{ }^{* * *} P<0.001 ;{ }^{* * * *} P<0.0001$. (C) Growth of GAS M49 strain 591/pFW11_glyluc2 in CDM without serine or supplemented with different concentrations $(0.01-10 \mathrm{mM})$ of serine, $n=3$. (D) Luciferase activity over time in GAS M49 strain 591/pFW11_gly/uc2 during growth without serine or in the presence of different concentrations of serine, as indicated, $n=3$. (E) Growth of GAS M49 strain 591/pFW11_glyluc2 in CDM without alanine or supplemented with different concentrations (0.01-10 mM) of alanine, $n=3$. (F) Luciferase activity over time in GAS M49 strain 591/pFW11_gly/uc2 during growth without alanine or in the presence of different concentrations of alanine, as indicated, $n=3$. Data are presented as mean values \pm standard deviation.

with the luc reporter gene assay and RT-qPCR results, expression of the gene was repressed under these conditions. In the presence of $0.1 \mathrm{mM}$ glycine, a $3 \mathrm{~kb}$ band could be detected with the ribogly and the Na+/Ala symp probes. The apparent size of the band corresponds to the calculated size of a co-transcript of the SAF gene and the cation efflux system gene (3113 bp, Figure 1). A second band with the apparent size of 600 bp could be detected with both probes. This corresponds to the product of termination within the SAF gene at the putative terminator TERM 125 (TransTermHP v2.07) (Kingsford et al., 2007). A 300 bp band was detected using the ribogly probe but not following hybridization with the $\mathrm{Na}+/$ Ala symp probe. The transcripts detected by Northern blotting are depicted schematically in relation to the corresponding genes (Figure 1).

\section{SAF Gene Transcript Stability Is Reduced at High Glycine Concentrations in the Medium}

Transcripts of genes controlled by riboswitches are often processed or exhibit differential stability (Mellin and Cossart,
2015). We aimed to investigate ribogly and SAF gene transcript stability under repressing and non-repressing conditions. WT GAS M49 strain 591 was grown in the presence of 0.1 or $10 \mathrm{mM}$ glycine, respectively. Transcription was stopped by addition of rifampicin and samples were collected for RT-qPCR analyses at 1, 2, 5, and 10 min following rifampicin treatment (Figure 5). 5S rRNA and groES mRNA served as controls. 5S rRNA was stable over the observation period. The half-life of groES mRNA was similar during growth in $0.1 \mathrm{mM}$ glycine $(1.8 \mathrm{~min})$ and $10 \mathrm{mM}$ glycine $(1.9 \mathrm{~min})$. Stability of ribogly was low under both conditions. Its half-life was $0.4 \mathrm{~min}$ in 0.1 and $10 \mathrm{mM}$ glycine. In contrast, the stability of $\mathrm{Na}+/$ Ala symp was low during growth in $10 \mathrm{mM}$ glycine $(0.5 \mathrm{~min})$ but much higher in $0.1 \mathrm{mM}$ glycine (5 min), suggesting an additional ribonuclease-dependent regulation level.

\section{ribogly Belongs to the Tandem Aptamer Glycine Riboswitches}

In the reference genome of $S$. pyogenes NZ131 (accession number: NC_011375.1), a 90 bp putative glycine riboswitch 


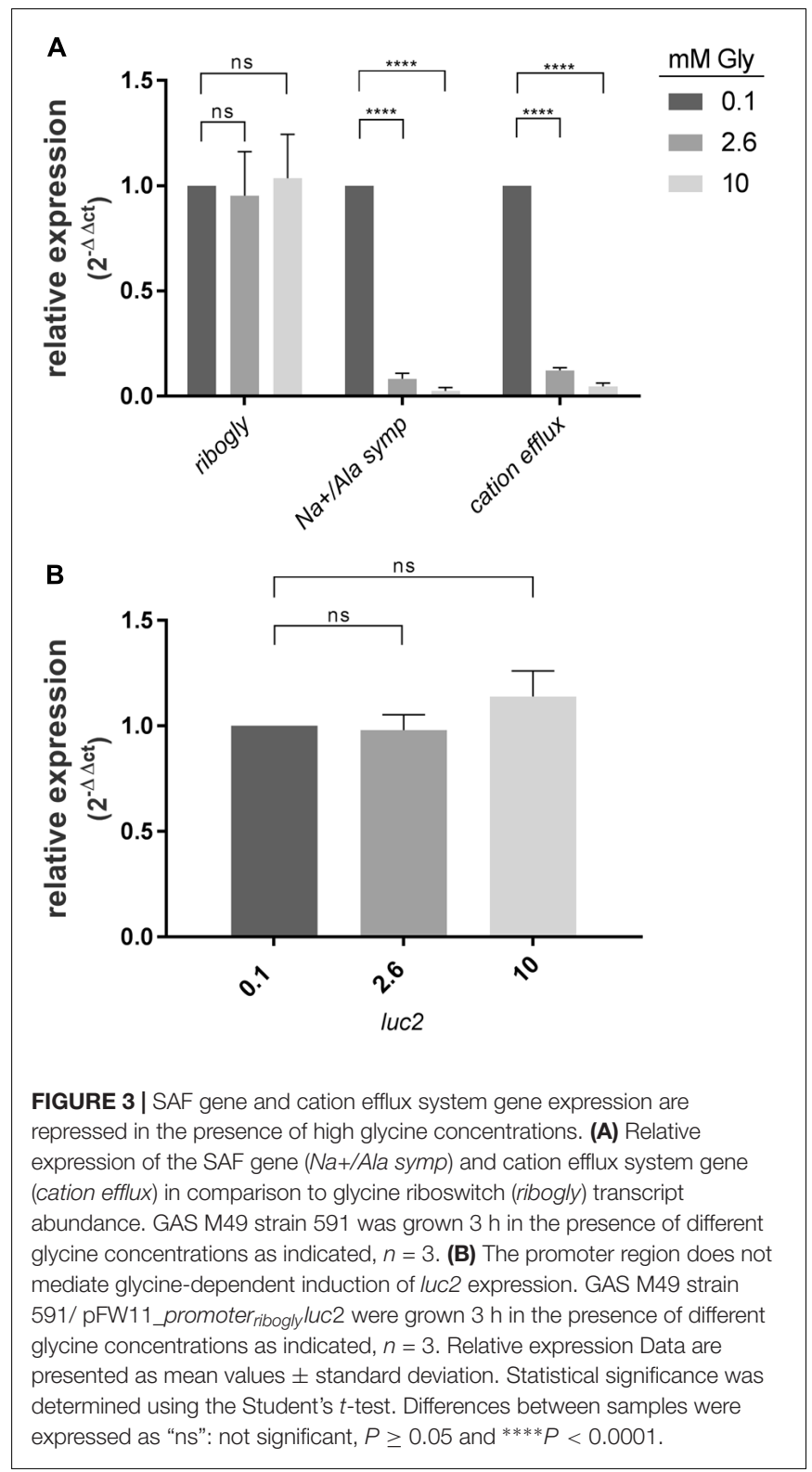

was annotated. A search using the rfam database (Nawrocki et al., 2015) predicted the presence of one glycine-binding aptamer. Since we observed a band of approximate $200 \mathrm{bp}$ in Northern blot analyses of total RNA from GAS M49 strain 591 (Figure 4), we performed secondary structure prediction using RNAfold (The ViennaRNA Web Services ${ }^{1}$ ). Secondary structure modeling was based on $182 \mathrm{bp}$ of the reference genome of $S$. pyogenes NZ131. The result is illustrated in Figure 6 using VARNA GUI (Darty et al., 2009). The analysis revealed the typical consensus structure of a tandem aptamer riboswitch containing two glycine binding sites (Esquiaqui et al., 2014; Ruff et al., 2016). The conserved residues are labeled in green.

${ }^{1}$ http://rna.tbi.univie.ac.at/

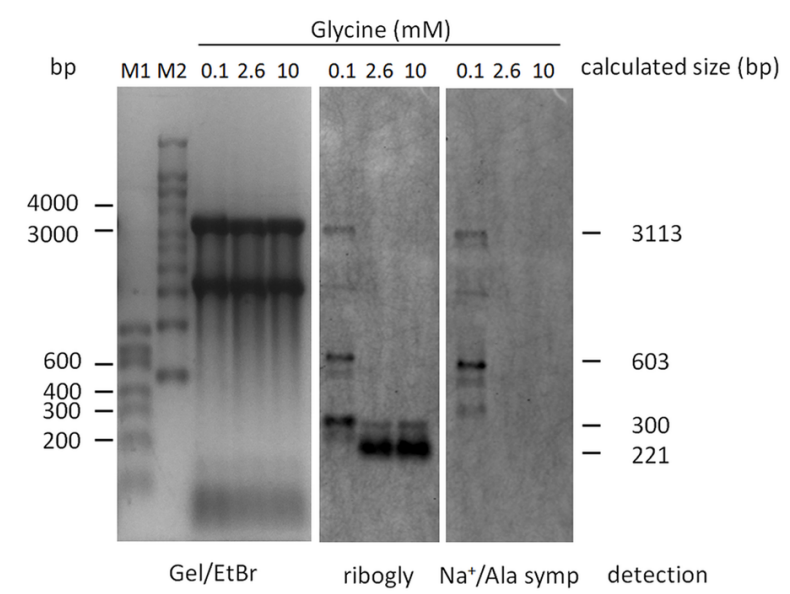

FIGURE 4 | Northern blot analyses. RNA was extracted from bacteria grown in $0.1,2.6$, and $10 \mathrm{mM}$ glycine as indicated. RNAs were separated by denaturing agarose gel electrophoreses and subsequently blotted onto a nylon membrane. The membrane was hybridized with probes specific for the glycine riboswitch (ribogly), stripped, and hybridized with a probe specific for the SAF gene (Na+/Ala symp). The ethidium bromide stained gel served as loading control.

\section{DISCUSSION}

Riboswitches often regulate expression of genes involved in the metabolism or transport of their ligands. Typically, glycine riboswitches induce the expression of genes responsible for glycine cleavage or export following glycine binding (Barrick et al., 2004). In Bacillus subtilis, the glycine riboswitch is composed of two similar aptamers followed by a single expression platform (Mandal et al., 2004). In S. pyogenes, one aptamer, similar to the B. subtilis aptamers, was predicted upstream of a putative SAF gene. Previously, we observed expression of the riboswitch region in GAS M49 strain 591 by intergenic tiling arrays (Patenge et al., 2012). In the SF370 clinical isolate, expression of the putative streptococcal riboswitch was detected by Northern blot analyses (Le Rhun et al., 2015). In this work, we investigated whether the putative glycine riboswitch mediates glycine-dependent gene regulation.

LUC reporter gene assays and Northern blot analyses revealed that expression of the SAF gene was repressed in the presence of glycine. The corresponding promoter region did not mediate glycine-dependent downregulation of $l u c$ in a promoter-reporter gene fusion construct. As a general rule, amino acid riboswitches increase the expression of glycine cleavage system genes or glycine export protein genes upon binding to glycine (Serganov and Patel, 2009; Serganov and Nudler, 2013). In Streptomyces griseus a detoxification system is activated by a glycine riboswitch (Tezuka and Ohnishi, 2014). In contrast, lysC in B. subtilis is repressed by a lysine-responsive riboswitch due to transcription termination in the presence of a high L-lysine concentration (Phan and Schumann, 2009). The lysC gene of B. subtilis consists of two overlapping reading frames coding for the $\alpha$ and $\beta$-subunits of a lysine-responsive aspartokinase II, which 

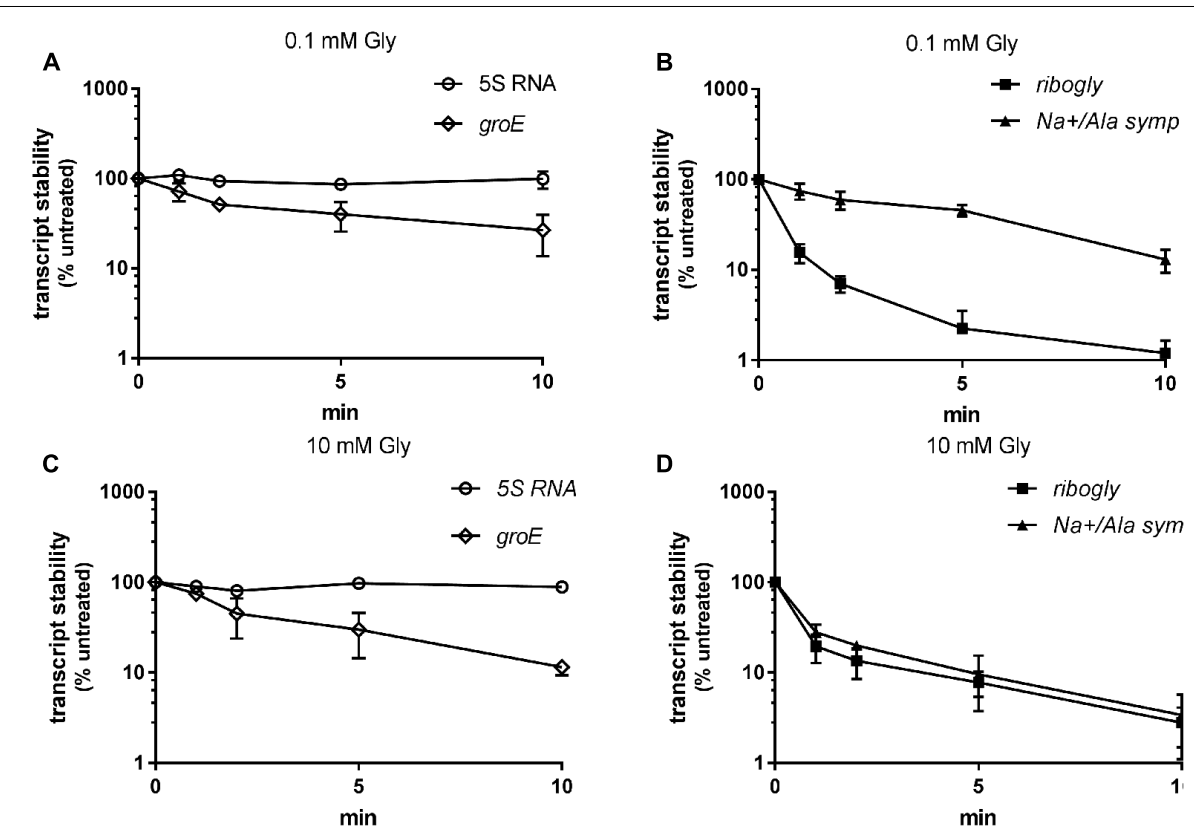

FIGURE 5 | Transcript stability under low $(0.1 \mathrm{mM})$ and high $(10 \mathrm{mM})$ glycine conditions. Stability of the glycine riboswitch (ribogly) and the SAF gene (Na+/Ala symp) as determined by RT-qPCR following treatment of the culture with rifampicin, $n=3.5 \mathrm{~S}$ rRNA and groES mRNA served as controls. Stability is presented as percent transcript level relative to time-point zero. (A) 5 S rRNA and groES mRNA stability at $0.1 \mathrm{mM}$ glycine. (B) ribogly and Na+/Ala symp mRNA stability at $0.1 \mathrm{mM}$ glycine. (C) $5 S$ rRNA and groES mRNA stability at $10 \mathrm{mM}$ glycine. (D) ribogly and Na+/Ala symp mRNA stability at $10 \mathrm{mM}$ glycine. The data are presented as the mean values \pm standard deviation.

catalyzes the first step in the biosynthesis of methionine, lysine, and threonine (Chen et al., 1987). The SAF gene in S. pyogenes encodes a member of the Alanine or Glycine:Cation Symporter (AGCS) Family [Transporter Classification Database (TCDB)] (Saier et al., 2016). Proteins belonging to the AGCS family have been reported to transport alanine and/or glycine in symport with $\mathrm{Na}^{+}$and/or $\mathrm{H}^{+}$. The dagA gene from the marine bacterium Alteromonas haloplanktis is a sodium-dependent transporter and is involved in the uptake of glycine and glutamine (MacLeod and MacLeod, 1992). We hypothesize that binding of glycine by the riboswitch in S. pyogenes leads to the downregulation of a so far unidentified glycine uptake system.

The truncated form of the $B$. subtilis riboswitch RNA controlling $l y s C$ is predominantly produced, even under conditions promoting anti-termination (Phan and Schumann, 2009). In contrast, we did only detect small amounts of the truncated $200 \mathrm{bp}$ riboswitch RNA under inducing conditions. Stability of the riboswitch RNA was low at all glycine concentrations tested. The half-life of the full-length transcript decreased at high glycine concentrations. A combination of classical riboswitch function and mRNA stability control has been reported recently. In $E$. coli, lys $C$ expression is controlled on the level of translation initiation by a lysine riboswitch. Upon lysine binding, the riboswitch adopts a conformation that sequesters the ribosomal binding site and at the same time exposes RNaseE cleavage sites (Caron et al., 2012). RNAse E is a component of the RNA degradosome in Gram negative bacteria (Callaghan et al., 2004). In most Gram positive organisms, RNaseE is replaced by other ribonucleases, e.g., RNaseY and

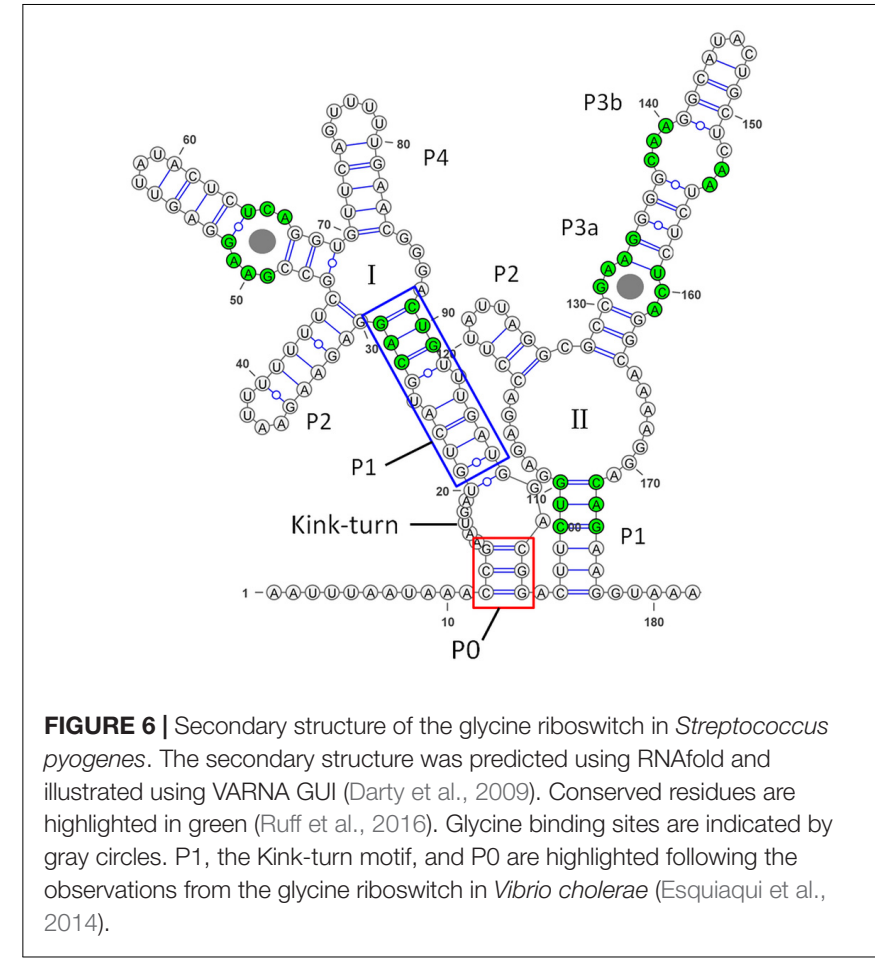

RNases J1 and J2 (Cho, 2017). In S. pyogenes, RNAse Y is involved in mRNA turnover (Chen et al., 2013). Structural investigation of the GlmS ribozyme from $B$. anthracis revealed 
autocatalytic cleavage of GlmS mRNA at a single site $5^{\prime}$ of the riboswitch sequence following binding of glucosamine-6phosphate (GlcN6P). Specific cleavage renders GlmS mRNA susceptible to degradation by RNase J (Cochrane et al., 2007). It is tempting to speculate that processing of the glycine riboswitch controlled transcript in S. pyogenes leads to the exposure of RNase cleavage sites and thereby to decreasing SAF gene transcript stability.

The glycine riboswitch is the only riboswitch that exhibits tandem architecture, with two adjacent, homologous aptamers followed by a single expression platform. In B. subtilis, glycine binding by the riboswitch aptamers has been initially reported to function in a cooperative manner (Mandal et al., 2004). However, a full-length derivative of the riboswitch containing its extended 5 ' leader, did not show cooperative binding (Sherman et al., 2012). The tandem glycine riboswitch from Vibrio cholerae, including the leader sequence, was studied using an equilibrium dialysisbased assay. The results showed that ligand binding by aptamer-1 is linked to aptamer dimerization and stabilizes the P1 stem of aptamer-2, which controls the expression platform (Ruff and Strobel, 2014). In a recent study, analysis of sequenced genomes revealed a significant number of singlet glycine riboswitches. Several singlet riboswitches were characterized biochemically and it could be demonstrated that singlet riboswitches were able to bind glycine with affinities comparable to those of previously published tandem glycine riboswitches. Conserved stem-loop structures (ghost aptamers), situated up- or down-stream of the singlet aptamer, respectively, form interactions with the aptamer domain that are necessary for ligand-binding activity (Ruff et al., 2016). In S. pyogenes, one aptamer was annotated upstream of the SAF gene. A second aptamer containing a P4 stem was revealed by in silico secondary structure predictions. The sequence directly upstream of the first aptamer is similar to the conserved leader region, which interacts with the recently discovered K-turn linker of tandem glycine riboswitches and modulates ligand binding (Kladwang et al., 2012; Sherman et al., 2012; Baird and FerreD'Amare, 2013; Esquiaqui et al., 2016). This indicates that the $S$. pyogenes glycine riboswitch belongs to the class of tandem riboswitches, featuring the recently identified K-turn linker.

Inducible expression systems are useful tools in molecular biology. Characterization of essential genes and production of potential toxic gene products are only two of many examples for the requirement of conditional gene expression control. Riboswitch based systems for precise gene regulation have the advantage, that expression can be controlled by the addition of small compounds that easily enter the cell and that are in many cases comparably inexpensive (Machtel et al., 2016). A glycine-inducible expression system has been developed using the riboswitch from B. subtilis. The authors could demonstrate glycine-dependent production

\section{REFERENCES}

Baird, N. J., and Ferre-D'Amare, A. R. (2013). Modulation of quaternary structure and enhancement of ligand binding by the K-turn of tandem glycine riboswitches. RNA 19, 167-176. doi: 10.1261/rna.036269.112 of recombinant proteins (Phan and Schumann, 2007). With a comparable strategy, a gene knock-down or knock-out system could be engineered, employing the $S$. pyogenes glycine riboswitch.

Riboswitches are potential targets for antimicrobial therapies (Machtel et al., 2016). Extensively studied targets include purine riboswitches. Purines are essential for bacterial survival and purine riboswitches control purine metabolism and transport (Lunse et al., 2014). There is an ongoing search for suitable purine analogs that bind the riboswitch with comparable affinity as guanosine (Kim et al., 2009; Mulhbacher et al., 2010). Beside essential pathways, riboswitch control of biofilm formation is a promising target for antimicrobial drugs (Reyes-Darias and Krell, 2017). Lysine riboswitches have also been explored as potential targets and several lysine analogs with high affinity binding have been identified, but there were difficulties arising from toxicity of the compounds and bacterial resistance (Blount et al., 2007). Glycine is required for optimal S. pyogenes growth. The $S$. pyogenes glycine riboswitch is highly conserved among different serotypes. Sequence identity of the riboswitch within the completed S. pyogenes genomic sequences is $99-100 \%$. Downregulation of streptococcal glycine transport by targeting the glycine riboswitch with a glycine analog could serve as a novel therapeutic strategy. Alternatively, ligands for the not yet characterized riboswitches in S. pyogenes should be identified and investigated for their drug target potential.

\section{AUTHOR CONTRIBUTIONS}

AK and NIP performed the experiments presented in the manuscript. AK, NIP, BK, and NAP contributed to the design of this study, analyses, and interpretation of the data, drafting the manuscript, and approved it for publication.

\section{FUNDING}

Our work was supported by grants from the Bundesministerium für Bildung und Forschung (FKZ 0315437B) and from the Ministerium für Bildung, Wissenschaft und Kultur, Mecklenburg-Vorpommern (ESF/14-BM-A55-0010/16), and the University Medicine Rostock (Forun 889008).

\section{SUPPLEMENTARY MATERIAL}

The Supplementary Material for this article can be found online at: https://www.frontiersin.org/articles/10.3389/fmicb. 2018.00200/full\#supplementary-material

Barrick, J. E., and Breaker, R. R. (2007). The distributions, mechanisms, and structures of metabolite-binding riboswitches. Genome Biol. 8:R239. doi: 10.1186/gb-2007-8-11-r239

Barrick, J. E., Corbino, K. A., Winkler, W. C., Nahvi, A., Mandal, M., Collins, J., et al. (2004). New RNA motifs suggest an expanded scope for riboswitches 
in bacterial genetic control. Proc. Natl. Acad. Sci. U.S.A. 101, 6421-6426. doi: $10.1073 /$ pnas. 0308014101

Batey, R. T. (2012). Structure and mechanism of purine-binding riboswitches. Q. Rev. Biophys. 45, 345-381. doi: 10.1017/S0033583512000078

Blount, K. F., Wang, J. X., Lim, J., Sudarsan, N., and Breaker, R. R. (2007). Antibacterial lysine analogs that target lysine riboswitches. Nat. Chem. Biol. 3, 44-49. doi: $10.1038 /$ nchembio842

Callaghan, A. J., Aurikko, J. P., Ilag, L. L., Gunter, G. J., Chandran, V., Kuhnel, K., et al. (2004). Studies of the RNA degradosome-organizing domain of the Escherichia coli ribonuclease RNase E. J. Mol. Biol. 340, 965-979. doi: 10.1016/j. jmb.2004.05.046

Caron, M. P., Bastet, L., Lussier, A., Simoneau-Roy, M., Masse, E., and Lafontaine, D. A. (2012). Dual-acting riboswitch control of translation initiation and mRNA decay. Proc. Natl. Acad. Sci. U.S.A. 109, E3444-E3453. doi: 10.1073/pnas. 1214024109

Chen, N. Y., Hu, F. M., and Paulus, H. (1987). Nucleotide sequence of the overlapping genes for the subunits of Bacillus subtilis aspartokinase II and their control regions. J. Biol. Chem. 262, 8787-8798.

Chen, Z., Itzek, A., Malke, H., Ferretti, J. J., and Kreth, J. (2013). Multiple roles of RNase $\mathrm{Y}$ in Streptococcus pyogenes mRNA processing and degradation. J. Bacteriol. 195, 2585-2594. doi: 10.1128/JB.00097-13

Cho, K. H. (2017). The structure and function of the gram-positive bacterial RNA degradosome. Front. Microbiol. 8:154. doi: 10.3389/fmicb.2017.00154

Cochrane, J. C., Lipchock, S. V., and Strobel, S. A. (2007). Structural investigation of the GlmS ribozyme bound to Its catalytic cofactor. Chem. Biol. 14, 97-105. doi: 10.1016/j.chembiol.2006.12.005

Dann, C. E. III, Wakeman, C. A., Sieling, C. L., Baker, S. C., Irnov, I., and Winkler, W. C. (2007). Structure and mechanism of a metal-sensing regulatory RNA. Cell 130, 878-892. doi: 10.1016/j.cell.2007.06.051

Darty, K., Denise, A., and Ponty, Y. (2009). VARNA: Interactive drawing and editing of the RNA secondary structure. Bioinformatics 25, 1974-1975. doi: 10.1093/bioinformatics/btp250

Esquiaqui, J. M., Sherman, E. M., Ionescu, S. A., Ye, J. D., and Fanucci, G. E. (2014). Characterizing the dynamics of the leader-linker interaction in the glycine riboswitch with site-directed spin labeling. Biochemistry 53, 3526-3528. doi: $10.1021 / \mathrm{bi500404b}$

Esquiaqui, J. M., Sherman, E. M., Ye, J. D., and Fanucci, G. E. (2016). Conformational flexibility and dynamics of the internal loop and helical regions of the kink-turn motif in the glycine riboswitch by site-directed spin-labeling. Biochemistry 55, 4295-4305. doi: 10.1021/acs.biochem.6b00287

Grundy, F. J., and Henkin, T. M. (1993). tRNA as a positive regulator of transcription antitermination in B. subtilis. Cell 74, 475-482. doi: 10.1016/00928674(93)80049-K

Grundy, F. J., Lehman, S. C., and Henkin, T. M. (2003). The L box regulon: lysine sensing by leader RNAs of bacterial lysine biosynthesis genes. Proc. Natl. Acad. Sci. U.S.A 100, 12057-12062. doi: 10.1073/pnas.2133705100

Kim, J. N., Blount, K. F., Puskarz, I., Lim, J., Link, K. H., and Breaker, R. R. (2009). Design and antimicrobial action of purine analogues that bind Guanine riboswitches. ACS Chem. Biol. 4, 915-927. doi: 10.1021/cb900146k

Kingsford, C. L., Ayanbule, K., and Salzberg, S. L. (2007). Rapid, accurate, computational discovery of Rho-independent transcription terminators illuminates their relationship to DNA uptake. Genome Biol. 8:R22. doi: 10.1186/ gb-2007-8-2-r22

Kladwang, W., Chou, F. C., and Das, R. (2012). Automated RNA structure prediction uncovers a kink-turn linker in double glycine riboswitches. J. Am. Chem. Soc. 134, 1404-1407. doi: 10.1021/ja2093508

Le Rhun, A., Beer, Y. Y., Reimegard, J., Chylinski, K., and Charpentier, E. (2015). RNA sequencing uncovers antisense RNAs and novel small RNAs in Streptococcus pyogenes. RNA Biol. 13, 177-195. doi: 10.1080/15476286.2015. 1110674

Levering, J., Fiedler, T., Sieg, A., van Grinsven, K. W., Hering, S., Veith, N., et al. (2016). Genome-scale reconstruction of the Streptococcus pyogenes M49 metabolic network reveals growth requirements and indicates potential drug targets. J. Biotechnol. 232, 25-37. doi: 10.1016/j.jbiotec.2016. 01.035

Lunse, C. E., Schuller, A., and Mayer, G. (2014). The promise of riboswitches as potential antibacterial drug targets. Int. J. Med. Microbiol. 304, 79-92. doi: 10.1016/j.ijmm.2013.09.002
Machtel, P., Bakowska-Zywicka, K., and Zywicki, M. (2016). Emerging applications of riboswitches - from antibacterial targets to molecular tools. J. Appl. Genet. 57, 531-541. doi: 10.1007/s13353-016-0341-x

MacLeod, P. R., and MacLeod, R. A. (1992). Identification and sequence of a $\mathrm{Na}(+)$-linked gene from the marine bacterium Alteromonas haloplanktis which functionally complements the dagA gene of Escherichia coli. Mol. Microbiol. 6, 2673-2681. doi: 10.1111/j.1365-2958.1992.tb01444.x

Mandal, M., Lee, M., Barrick, J. E., Weinberg, Z., Emilsson, G. M., Ruzzo, W. L., et al. (2004). A glycine-dependent riboswitch that uses cooperative binding to control gene expression. Science 306, 275-279. doi: 10.1126/science.110 0829

Mellin, J. R., and Cossart, P. (2015). Unexpected versatility in bacterial riboswitches. Trends Genet. 31, 150-156. doi: 10.1016/j.tig.2015.01.005

Mulhbacher, J., Brouillette, E., Allard, M., Fortier, L. C., Malouin, F., and Lafontaine, D. A. (2010). Novel riboswitch ligand analogs as selective inhibitors of guanine-related metabolic pathways. PLoS Pathog. 6:e1000865. doi: 10.1371/ journal.ppat. 1000865

Nawrocki, E. P., Burge, S. W., Bateman, A., Daub, J., Eberhardt, R. Y., Eddy, S. R., et al. (2015). Rfam 12.0: updates to the RNA families database. Nucleic Acids Res. 43, D130-D137. doi: 10.1093/nar/gku1063

Patenge, N., Billion, A., Raasch, P., Normann, J., Wisniewska-Kucper, A., Retey, J., et al. (2012). Identification of novel growth phase- and media-dependent small non-coding RNAs in Streptococcus pyogenes M49 using intergenic tiling arrays. BMC Genomics 13:550. doi: 10.1186/1471-2164-13-550

Phan, T. T., and Schumann, W. (2007). Development of a glycine-inducible expression system for Bacillus subtilis. J. Biotechnol. 128, 486-499. doi: 10.1016/ j.jbiotec.2006.12.007

Phan, T. T., and Schumann, W. (2009). Transcriptional analysis of the lysineresponsive and riboswitch-regulated lysC gene of Bacillus subtilis. Curr. Microbiol. 59, 463-468. doi: 10.1007/s00284-009-9461-4

Podbielski, A., Woischnik, M., Leonard, B. A., and Schmidt, K. H. (1999). Characterization of $n r a$, a global negative regulator gene in group A streptococci. Mol. Microbiol. 31, 1051-1064. doi: 10.1046/j.1365-2958.1999. 01241.x

Reizer, J., Reizer, A., and Saier, M. H. Jr. (1994). A functional superfamily of sodium/solute symporters. Biochim. Biophys. Acta 1197, 133-166. doi: 10.1016/ 0304-4157(94)90003-5

Reyes-Darias, J. A., and Krell, T. (2017). Riboswitches as potential targets for the development of anti-biofilm drugs. Curr. Top. Med. Chem. doi: 10.2174/ 1568026617666170407163517 [Epub ahead of print].

Rodionov, D. A., Vitreschak, A. G., Mironov, A. A., and Gelfand, M. S. (2003). Regulation of lysine biosynthesis and transport genes in bacteria: yet another RNA riboswitch? Nucleic Acids Res. 31, 6748-6757.

Ruff, K. M., Muhammad, A., McCown, P. J., Breaker, R. R., and Strobel, S. A. (2016). Singlet glycine riboswitches bind ligand as well as tandem riboswitches. RNA 22, 1728-1738. doi: 10.1261/rna.057935.116

Ruff, K. M., and Strobel, S. A. (2014). Ligand binding by the tandem glycine riboswitch depends on aptamer dimerization but not double ligand occupancy. RNA 20, 1775-1788. doi: 10.1261/rna.047266.114

Saier, M. H. Jr., Reddy, V. S., Tsu, B. V., Ahmed, M. S., Li, C., and Moreno-Hagelsieb, G. (2016). The transporter classification database (TCDB): recent advances. Nucleic Acids Res. 44, D372-D379. doi: 10.1093/nar/gkv 1103

Schmittgen, T. D., and Livak, K. J. (2008). Analyzing real-time PCR data by the comparative C(T) method. Nat. Protoc. 3, 1101-1108. doi: 10.1038/nprot. 2008.73

Serganov, A., and Nudler, E. (2013). A decade of riboswitches. Cell 152, 17-24. doi: 10.1016/j.cell.2012.12.024

Serganov, A., and Patel, D. J. (2009). Amino acid recognition and gene regulation by riboswitches. Biochim. Biophys. Acta 1789, 592-611. doi: 10.1016/j.bbagrm. 2009.07.002

Sherman, E. M., Esquiaqui, J., Elsayed, G., and Ye, J. D. (2012). An energetically beneficial leader-linker interaction abolishes ligand-binding cooperativity in glycine riboswitches. RNA 18, 496-507. doi: 10.1261/rna.031286.111

Sims, S. A., Colquhoun, S., Wyber, R., and Carapetis, J. R. (2016). "Global disease burden of group A Streptococcus," in Streptococcus Pyogenes: Basic Biology to Clinical Manifestations, eds J. J. Ferretti, D. L. Stevens, and V. A. Fischetti (Oklahoma City, OK: University of Oklahoma Health Sciences Center). 
Sudarsan, N., Lee, E. R., Weinberg, Z., Moy, R. H., Kim, J. N., Link, K. H., et al. (2008). Riboswitches in eubacteria sense the second messenger cyclic di-GMP. Science 321, 411-413. doi: 10.1126/science.1159519

Sudarsan, N., Wickiser, J. K., Nakamura, S., Ebert, M. S., and Breaker, R. R. (2003). An mRNA structure in bacteria that controls gene expression by binding lysine. Genes Dev. 17, 2688-2697. doi: 10.1101/gad.1140003

Tezuka, T., and Ohnishi, Y. (2014). Two glycine riboswitches activate the glycine cleavage system essential for glycine detoxification in Streptomyces griseus. J. Bacteriol. 196, 1369-1376. doi: 10.1128/JB.01480-13

van de Rijn, I., and Kessler, R. E. (1980). Growth characteristics of group A streptococci in a new chemically defined medium. Infect. Immun. 27, 444-448.

Winkler, W., Nahvi, A., and Breaker, R. R. (2002). Thiamine derivatives bind messenger RNAs directly to regulate bacterial gene expression. Nature 419, 952-956. doi: 10.1038/nature01145

Winkler, W. C., Cohen-Chalamish, S., and Breaker, R. R. (2002). An mRNA structure that controls gene expression by binding FMN. Proc. Natl. Acad. Sci. U.S.A. 99, 15908-15913. doi: 10.1073/pnas.212628899
Winkler, W. C., Nahvi, A., Sudarsan, N., Barrick, J. E., and Breaker, R. R. (2003). An mRNA structure that controls gene expression by binding S-adenosylmethionine. Nat. Struct. Biol. 10, 701-707. doi: 10.1038/nsb967

Yanofsky, C. (1981). Attenuation in the control of expression of bacterial operons. Nature 289, 751-758. doi: 10.1038/289751a0

Conflict of Interest Statement: The authors declare that the research was conducted in the absence of any commercial or financial relationships that could be construed as a potential conflict of interest.

Copyright (c) 2018 Khani, Popp, Kreikemeyer and Patenge. This is an open-access article distributed under the terms of the Creative Commons Attribution License (CC BY). The use, distribution or reproduction in other forums is permitted, provided the original author(s) and the copyright owner are credited and that the original publication in this journal is cited, in accordance with accepted academic practice. No use, distribution or reproduction is permitted which does not comply with these terms. 